\title{
THE EFFECTIVE BANDWIDTH OF A SYSTEM*
}

\author{
By PAUL M. CHIRLIAN (Stevens Institute of Technology)
}

Summary. Bounds on the effective bandwidth, based upon transient response are obtained.

1. Introduction. The use of a numerical bandwidth is an attempt to describe a system frequency response by a single number. This is in general only useful when the frequency response curves are of similar shape. However, very often useable results are obtained. The criterion used to determine the bandwidth depends upon the device. A definition will be discussed here that is useful when transient response is important. We shall consider the bandwidth to include all frequencies which make a substantial contribution to the (transient) response. That is if the difference between the actual time response and the time response calculated by assuming that there are no frequency components above $\omega_{c}$ is negligible, then $\omega_{c}$ is an upper bound on the bandwidth. The unit step response shall be used as a standard here. If a different time function was used different results could be obtained.

In general this definition of bandwidth requires that lengthy transient response calculations be made. To avoid this we shall develop bounds on $\omega_{c}$ which can be very easily obtained.

2. Bandwidth bounds. We shall consider that $H(\omega)$, the system function is normalized so that the final value of the unit step response is unity. (We are only considering high frequency response here.) Consider that $A(t)$ is the actual unit step response and $A_{1}(t)$ is the one which is calculated assuming that $H(\omega)=0$ for $\omega>\omega_{c}$. The percent error is

$$
|\% \delta(t)|=\left|A(t)-A_{1}(t)\right| \times 100 .
$$

Now let us introduce the bounds. If, we have a function $G(\omega)$ such that

$$
0 \leq|H(\omega)| \leq G(\omega) \text { for } \omega>\omega_{c}
$$

then [1]

$$
|\% \delta(t)| \leq \frac{100}{\pi} \int_{\omega_{e}}^{\infty} \frac{G(\omega)}{\omega} d \omega .
$$

Then in determining a criterion for the bandwidth we choose a $\% \delta$ to use. Let us call this $\% \delta_{b}$ then obtain an $\omega_{c}$ such that

$$
\% \delta_{b}=\frac{100}{\pi} \int_{\omega 。}^{\infty} \frac{G(\omega)}{\omega} d \omega
$$

is satisfied. This $\omega_{c}$ is an upper bound on the bandwidth.

To simplify our calculation let us assume that

$$
|H(\omega)| \leq \epsilon\left(\omega_{0} / \omega\right)^{n}, \quad \omega \geq \omega_{0},
$$

substituting in (4) we obtain (note that $\omega_{c}>\omega_{0}$ )

$$
\omega_{c}=[100 \epsilon / \pi n \% \delta]^{1 / n} \omega_{0} .
$$

*Received December 16, 1966. 
For instance if $\% \delta=10 \%, n=3$, and $\epsilon=1.0$ then $\omega_{c}=1.02 \omega_{0}$. This is almost at the frequency where $H(\omega)$ starts to fall off. On the other hand if $n=1$ then $\omega_{c}=3.183 \omega_{0}$.

Another type of asymptotic fall off may be useful in distributed integrated circuits.

In this case

$$
0 \leq|H(\omega)| \leq \epsilon \exp \left(-n\left(\omega / \omega_{0}\right)^{1 / 2}\right) .
$$

Then substituting in (4) we obtain

$$
\% \delta_{b}=\frac{-200 \epsilon}{\pi} E i\left(-n\left(\omega_{c} / \omega_{0}\right)^{1 / 2}\right)
$$

where

$$
\operatorname{Ei}(-x)=-\int_{z}^{\infty}\left(e^{-y} / y\right) d y
$$

this is a form of the exponential integral and is tabulated [2]. If $E i(-x)=-y$ then

$$
E i^{-1}(-y)=-x
$$

Then

$$
\omega_{c}=\frac{+\omega_{0}}{n^{2}}\left\{E i^{-1}\left(-\% \delta_{b} \pi / 200 \epsilon\right)\right\}^{2} .
$$

These values can be obtained from the table [2].

3. Conclusion. We have defined bandwidth in terms of transient response. The frequency bandwidth includes those frequencies which are important to the transient response. Such a bandwidth is not only useful as a figure of merit it also enables the system designer to specify frequency requirements in a cascade of equipment. As a figure of merit a relatively large value of $\% \delta_{b}$ should be chosen. (e.g. it would be unrealistic to base the bandwidth on $a \% \delta_{b}=0.001$ ). On the other hand if $\omega_{c}$ is used to ascertain the bandwidth of devices that are to be cascaded with the one in question then a $\% \delta_{b}$ based upon desired system response should be used. In this case $\delta_{b}$ may be small.

\section{REFERENCES}

[1] P. M. Chirlian, Bounds on the error in the unit step response of a network, Quart. Appl. Math. 16, 432-436 (1959)

[2] E. Jahnke, F. Emde, F. Losch, Tables of higher functions, McGraw-Hill, New York, 1960, pp. 17-25 